\title{
Thyroid Hormone-regulated Brain Mitochondrial Genes Revealed by Differential cDNA Cloning
}

Elena Vega-Núñez, ${ }^{*}$ Ana Menéndez-Hurtado, ${ }^{*}$ Rafael Garesse, ${ }^{\ddagger}$ Angel Santos, ${ }^{\S}$ and Ana Perez-Castillo*

${ }^{*}$ Instituto de Investigaciones Biomédicas, C.S.I.C., 28029-Madrid, Spain; ${ }^{\ddagger}$ Departamento de Bioquímica, UAM-Instituto de Investigaciones Biomédicas, 28029-Madrid, Spain; and 'Departamento de Bioquímica y Biología Molecular, Fac. Medicina, UCM, 28040-Madrid, Spain

\begin{abstract}
Thyroid hormone $\left(\mathrm{T}_{3}\right)$ plays a critical role in the development of the central nervous system and its deficiency during the early neonatal period results in severe brain damage. However the mechanisms involved and the genes specifically regulated by $T_{3}$ during brain development are largely unknown. By using a subtractive hybridization technique we have isolated a number of cDNAs that represented mitochondrial genes (12S and 16S rRNAs and cytochrome $c$ oxidase subunit III). The steady state level of all three RNAs was reduced in hypothyroid animals during the postnatal period and $T_{3}$ administration restored control levels. During fetal life the level of 16S rRNA was decreased in the brain of hypothyroid animals, suggesting a prenatal effect of thyroid hormone on brain development. Since $T_{3}$ does not affect the amount of mitochondrial DNA, the results suggest that the effect of $T_{3}$ is at transcriptional and/or postranscriptional level. In addition, the transcript levels for two nuclear-encoded mitochondrial cytochrome $c$ oxidase subunits: subunits IV and VIc were also decreased in the brains of hypothyroid animals. Hypothyroidism-induced changes in mitochondrial RNAs were followed by a concomitant $40 \%$ decrease in cytochrome $c$ oxidase activity. This study shows that $T_{3}$ is an important regulator of mitochondrial function in the neonatal brain and, more importantly, provides a molecular basis for the specific action of this hormone in the developing brain. (J. Clin. Invest. 1995. 96:893-899.) Key words: hypothyroidism - development • central nervous system • mitochondrial function • nuclear receptors
\end{abstract}

\section{Introduction}

In vertebrates, thyroid hormone $\left(T_{3}\right)^{1}$ is essential for many fundamental processes, including normal development, growth,

Address correspondence to Ana Perez-Castillo, Instituto de Investigaciones Biomédicas (CSIC), Arturo Duperier, 4, 28029-Madrid, Spain. Phone: 34-1-585-4625; FAX: 34-1-585-4587; or to Angel Santos, Departamento de Bioquimica y Biologia Molecular, Fac. Medicina, UCM, 28040-Madrid, Spain. Phone: 34-1-394-1680; FAX:34-1-394-1691.

Received for publication 15 December 1994 and accepted in revised form 10 April 1995.

1. Abbreviations used in this paper: COX, cytochrome $c$ oxidase; mt, mitochondrial; ND4, NADH dehydrogenase subunit 4; nt, nucleotide; $\mathrm{T}_{3}$, thyroid hormone; TR, thyroid hormone receptor; $\mathrm{Tx}$, hypothyroid.

J. Clin. Invest.

(C) The American Society for Clinical Investigation, Inc.

0021-9738/95/08/0893/07 \$2.00

Volume 96, August 1995, 893-899 and metabolism (1-3). Thyroid hormone is also known to exert dramatic morphological, biochemical, and physiological changes on amphibian metamorphosis (4). These actions are primarily exerted on the genome after $T_{3}$ binds to specific nuclear receptors (TR) that activate or suppress thyroid hormoneresponsive genes (5). Several isoforms of TRs have been isolated and shown to be members of a superfamily of ligandactivated transcriptional regulatory proteins, sharing a highly conserved DNA-binding domain and less well conserved $\mathrm{COOH}$-terminal regions required for ligand binding. The enhancement of promoter activity by TR requires a sequence specific interaction of the receptor with a thyroid hormone response element $(5,6)$. TRs are believed to function as homodimers or as heterodimers with proteins such as retinoid $\mathrm{X}$ receptors (7).

It is known that adequate thyroid hormone levels are absolutely required for normal development of the central nervous system. Thyroid hormone deficiency during development leads to growth abnormalities and irreversible mental retardation (4, 8 ). In humans the syndrome, called cretinism, includes deafmutism, short stature, and profound mental retardation. Experimentally induced hypothyroidism in rats results in numerous abnormalities, including reduction in dendritic arborization of cerebellar Purkinje cells, reduction of neuronal outgrowth, poor connectivity among neurons, changes in microtubule content and impaired myelin deposition, cell migration, and synaptogenesis (8). In the rat, normal brain development requires the presence of $T_{3}$ during the period between 10 and $15 \mathrm{~d}$ after birth and its deficiency during the early neonatal period results in severe brain damage $(8,9)$. This is an important period for neuronal differentiation and maturation and during the first 30 d postpartum a rapid myelogenesis and intense glial proliferation and development of neuronal processes take place (10). We have observed that during this period both the number of $T_{3}$ receptors and the amount of receptor mRNA $(11,12)$ rise together with the level of $T_{3}$ in brain. Little is known however of the genomic targets for ligand-activated TR in this complex biological program. In this context, we have recently shown that the early gene NGFI-A, which is thought to be implicated in proliferation and differentiation processes, is highly regulated by thyroid hormone in the developing brain $(13,14)$. In tissues different from the brain including heart, kidney, liver, and skeletal muscle, $T_{3}$ regulation can be explained, at least in part, by a control over mitochondrial function. However, although the neurologic symptoms displayed by hypothyroid patients resemble, in part, those caused by mitochondrial dysfunction, there have been contradictory data regarding a role of $T_{3}$ in the mitochondria of the central nervous system $(15,16)$.

To identify genes whose expression is dependent on thyroid hormone during brain development, we have used a subtractive hybridization technique to isolate cDNA clones whose cognate messengers are expressed more abundantly in euthyroid brain. 
We have isolated several cDNA clones, three of which code for known mitochondrial genes: $12 \mathrm{~S}$ and $16 \mathrm{~S}$ mitochondrial (mt) rRNAs and the subunit III of cytochrome $c$ oxidase (COX III). In accordance with the changes in the levels of these RNAs, the specific activity of the inner membrane enzyme cytochrome $c$ oxidase was also reduced in hypothyroid animals when contrasted with age-matched controls. Our data also show a biological action for $T_{3}$ even during the gestation period. The results presented here are direct evidence that removal of $T_{3}$ produces specific deficits in the brain mitochondria of neonatal rats and provide a molecular basis for the specific action of this hormone in the developing rat brain.

\section{Metheds}

Animal treatment. To induce fetal and neonatal hypothyroidism, dams were given $0.02 \%$ methylmercaptoimidazole (MMI) in drinking water at day 9 of pregnancy. MMI administration was continued throughout the lactating period. On postnatal day 5 pups were surgically thyroidectomized. Adult hypothyroidism was induced with MMI treatment for 3 wk. With these treatments, cytosolic $T_{3}$ was below the level of detectability. For the time-course experiments, 12-15-d-old hypothyroid (Tx) rats were injected intraperitoneally with $\mathrm{T}_{3}(200 \mu \mathrm{g} / 100$ gram of body weight, once daily) and killed at the indicated times. Control animals received saline injections. Animals were killed at different ages by decapitation and the tissues were rapidly removed, dissected, and frozen.

Subtractive hybridization. $2 \mu \mathrm{g}$ of poly(A) ${ }^{+}$RNA isolated from the cerebral cortex of 15-d-old euthyroid animals were used to generate an oligo(dT)-primed library in the plasmid vector Bluescribe M13+ (Stratagene Inc., LaJolla, CA), modified as in reference 17. For the synthesis of the subtracted probe, first strand cDNA was synthesized from poly $(\mathrm{A})^{+}$isolated from 15-d-old euthyroid cerebral cortex and mixed with an excess of poly $(A)^{+}$RNA extracted from the same brain region of 15-d-old hypothyroid rats. Selection for the unhybridized (single-stranded) cDNA was performed with hydroxyapatite (Bio-Rad Laboratories Inc., Brussels, Belgium) equilibrated with $0.12 \mathrm{M}$ sodium phosphate buffer ( $\mathrm{pH} \mathrm{6.8)}$ at $60^{\circ} \mathrm{C}$ in a water-jacketed column. Isolated single-stranded cDNA was then subjected to a second round of subtraction. The subtracted probe obtained was radiolabeled with $\left[\alpha-{ }^{32} \mathrm{P}\right] \mathrm{dCTP}$ to high specific activity by random priming and used to screen 120,000 clones from the euthyroid library. Clones representing putative thyroid hormone-regulated cDNAs were then differentially screened. cDNA clones picked up in this secondary screening were restricted to release the cDNA inserts and run on agarose gels. Gels were Southern-blotted onto duplicate nylon (Nytran; Renner GmbH, Dannstadt, Germany) membranes and again differentially screened. The putative positive clones were used for subsequent sequencing and expression analysis.

Northern blot analysis. Total RNA was extracted by homogenization in guanidinium thiocyanate as previously described (18). $10 \mu \mathrm{g}$ of RNA were electrophoresed on a $2.2-\mathrm{M}$ formaldehyde, $1 \%$ agarose gel in 1 $\times$ Mops buffer at $100 \mathrm{~V}$ for 3-4 $\mathrm{h}$ and transferred to nylon membranes. The different probes were generated using random primers $\left(>10^{8} \mathrm{cpm} /\right.$ $\mu \mathrm{g})$ and hybridized with the membranes for $20 \mathrm{~h}$ at $42^{\circ} \mathrm{C}$ (50\% formamide, $3 \times$ SSC, $0.2 \%$ SDS). COX IV and COX VIc cDNAs were obtained by PCR amplification as follows: COX IV, (forward primer: 5'-CAG CAG TGG CAG AAT GTT GG-3'; reverse primer: 5'-ATC AAA GGT ATG AGG GAT GGG G-3') that will amplify a fragment of 414 bp (nucleotide [nt] 59-473) and COX VIc, (forward primer: 5'-ATT GGG CTC ACT TCC TGC-3'; reverse primer: 5'-ACC CCA TCA AGC AGA ACC-3') that will amplify a fragment of 277 bp (nt 28-305). The washing conditions were $2 \times$ SSC and $0.5 \%$ SDS at $65^{\circ} \mathrm{C}$ for mild washing and then $0.2 \times \mathrm{SSC}$ and $0.5 \%$ SDS at $65^{\circ} \mathrm{C}$ for stringent washing. Methylene blue staining of the membranes was used as loading control. For quantification autoradiographs were scanned with a Silver Scanner densitometer (La Cie, Tualatin, OR) and the optical densities of each specific signal were normalized with the corresponding value obtained for the $28 \mathrm{~S}$ and $18 \mathrm{~S}$ rRNA. Values in the text are the mean of three different experiments corresponding to three different groups of animals.

DNA slot blot analysis. For DNA preparation, brains from two control and two hypothyroid animals were extracted after treatment with proteinase K and DNase-free RNase as previously described (19). Different amounts of total DNA were blotted to Zeta-probe filter (BioRad Laboratories Inc.) using the Manifold II system (Schleicher \& Schuel, Inc., Keene, N.H.) and fixed on the filter following the manufactures indications. To quantify the mtDNA, filters were probed at high stringency with an $\left[\alpha-{ }^{32} \mathrm{P}\right] \mathrm{dCTP}$-labeled rat 16S rRNA clone. After several washes with $0.1 \% \mathrm{SDS}, 0.1 \times \mathrm{SSC}$ at $68^{\circ} \mathrm{C}$ the filter was autoradiographed at different periods of time to be sure that the signal was in the linear range of response.

Determination of cytochrome $c$ oxidase activity. Control and hypothyroid brains from 15-d-old animals were homogenized in $5 \mathrm{ml}$ of 20 mM Tris-HCl, pH 8.0, 0.2 mM EDTA, 1 mM DTT, 10\% glycerol, and $1 \mathrm{mM}$ PMSF. COX activity was determined in the homogenate as previously described (20). Protein content was measured by the procedure of Bradford (21). The data correspond to the average of at least three determinations in each of two independent experiments. $P$ $<0.002$.

Statistical analysis. Statistical analysis were performed using Student's $t$ test. Values are reported as the mean \pm SD. $P$ values $\leq 0.05$ were considered significant.

\section{Results}

Cloning and expression of $T_{3}$-sensitive RNAs in developing rat brain. To identify genes whose expression is dependent on thyroid hormone during brain development, we used a subtractive hybridization technique. Since it is known that, in the developing rat brain the first 3 postnatal wk seem to represent a period of particular sensitivity to thyroid hormone (22), both the library and the subtracted probe were constructed from poly $(\mathrm{A})^{+}$RNA isolated from the cerebral cortex of 15 -d-old euthyroid neonates. Using this technique we identified, in addition to other cDNAs, three independent clones initially named $1 \mathrm{~B} 2(0.6 \mathrm{~kb}), 1 \mathrm{D} 6(1.0 \mathrm{~kb})$, and $2 \mathrm{~A} 9(0.85 \mathrm{~kb})$. Sequence analysis of these clones and search of the GenBank and EMBL databases revealed that they encoded the two mitochondrial rRNAs (12S and 16S) and the subunit III of cytochrome $c$ oxidase, respectively. The regions of the rat mitochondrial genome represented by these cDNAs are as follows: $12 \mathrm{~S}$ rRNA, from nt 70 to nt 685; 16S rRNA, from nt 1106 to nt 2098 and cytochrome $c$ oxidase, from nt 8638 to nt 9440 .

The effect of thyroid hormone on these genes was studied by Northern blot analysis of total brain RNA, extracted from control and hypothyroid animals at different ages. Early hypothyroidism markedly diminished the steady state level of all three RNAs observed in control animals, ranging between twoand fivefold reduction depending on the specific RNA and the age of the animal (Figs. 1 and 2). In general, this reduction can be observed throughout the whole neonatal period. Hypothyroidism induced in adult rats also caused a significant decrease on 12S rRNA levels (2.2-fold) although we did not observe significant changes in the concentration of both $16 \mathrm{~S}$ rRNA and COX III mRNA in these animals. After hormone administration, the content of 12S rRNA and COX III mRNA in hypothyroid 15-d-old animals slowly increased, reaching normal levels 48-72 $\mathrm{h}$ after treatment (Figs. 1 and 2). A similar pattern of response to $T_{3}$ was also found for 16S rRNA (data not shown).

Although it has been a matter of controversy during many 


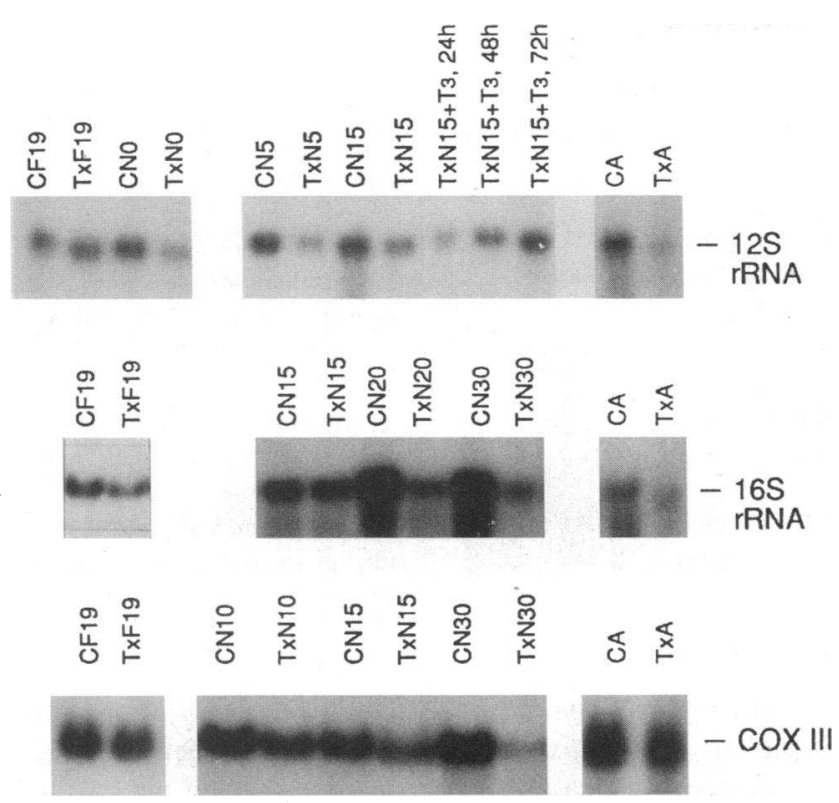

Figure 1. Thyroid hormone regulation of brain mitochondrial genes (12S rRNA, 16S rRNA, and COX III) during development. Northern analysis were performed as described in Methods. Representative autoradiograms are shown. F, fetuses; $N$, neonates; $A$, adult; $C$, control; $T x$ $+T_{3}, T x$ injected with a daily dose of $T_{3}(200 \mu \mathrm{g} / 100$ grams of body weight) and killed at the indicated times after the first injection.

years whether thyroid hormone could affect brain development before birth (23) and the generalized idea was that $T_{3}$ was not required during fetal life, our results show that thyroid hormone deficiency during development also led to changes in the expression of the $16 \mathrm{~S}$ gene in 19-d-old fetuses. Thus, as shown in Figs. 1 and 2, the brain of 19-d-old hypothyroid fetuses showed a twofold reduction in 16S rRNA levels. A similar reduction on 12S rRNA levels was also observed in hypothyroid N0, suggesting also a prenatal regulation of this gene by $\mathrm{T}_{3}$.

Since the genes coded in the mitochondrial genome are transcribed from a single promoter region (24), we further determined whether the control of $T_{3}$ over mtRNA was a generalized one. For this purpose, we measured the steady state levels of other representative mitochondrial-encoded transcripts: cytochrome $c$ oxidase subunit I (COX I), apocytochrome $b$ and NADH dehydrogenase subunit 4 (ND4), not picked up in our screening. As shown in Fig. 3, the levels of all of them were significantly reduced in hypothyroid animals, suggesting that $T_{3}$ is regulating the concentration of all mitochondrial transcripts.

Effect of thyroid hormone on mitochondrial gene expression in the liver. We next examined whether the effects of hypothyroidism upon brain mitochondrial gene expression during development were similar in other $T_{3}$ responsive tissues. In contrast with the situation in the brain, a significant decrease in $12 \mathrm{~S}$ rRNA was observed $12 \mathrm{~h}$ after $\mathrm{T}_{3}$ administration to 15 -d-old animals. No differences were observed in steady state levels of both $12 \mathrm{~S}$ and $16 \mathrm{~S}$ rRNAs in the liver of 15- and 30-d-old hypothyroid rats (Fig. 4). These results are in contrast with data reported by other authors showing no effect or even an stimulatory effect of $T_{3}$ on 12S rRNA levels in the adult liver $(25,26)$. In agreement with previous reports in adult rats $(27)$, the levels of COX III transcripts were slightly reduced in the liver of 15-d-old animals, and increased $48 \mathrm{~h}$ after hormone treatment to reach higher values than those found in euthyroid animals (2.5-fold). These results suggest important differences in the regulatory factors controlling these genes in the different tissues.

Effect of hypothyroidism on mtDNA content in the developing brain. To know if the decrease in mtRNAs observed in hypothyroid brain was due to a reduction in the number of mitochondria per cell, we next examined the effect of thyroid state on brain mtDNA content. Total DNA isolated from the
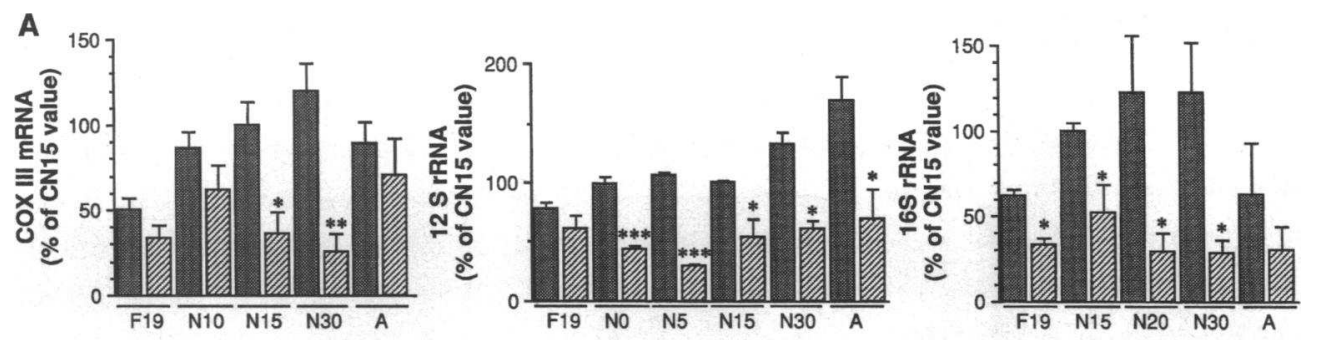

B



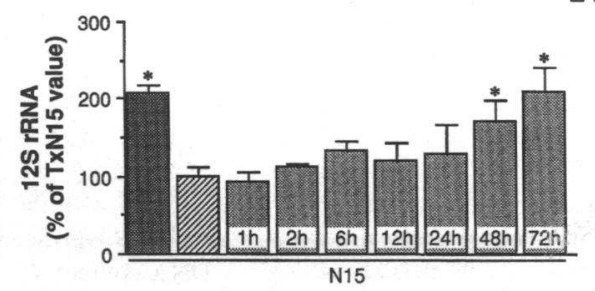

Figure 2. Content of 12S rRNA, $16 \mathrm{~S}$ rRNA, and COX III mRNA in control and hypothyroid brain during development. Steady state $(A)$ and kinetics analysis $(B)$ of 1B2 (12S rRNA), 1D6 (16S rRNA), and 2A9 (COX III) mRNAs were performed in control and hypothyroid animals of different ages. RNA levels were determined by densitometric analysis of three different Northern's corresponding to three different experimental groups. The hybridization signals were quantitated in a Silver Scanner densitometer and normalized to the methylene blue signal. Results are expressed as mean \pm SD. ${ }^{*} P \leq 0.05$ vs $C(A)$ or $\operatorname{Tx}(B) ; * * P \leq 0.01$ vs $C(A)$ or $\operatorname{Tx}(B) .{ }^{* * * P} \leq 0.001$ vs $\mathrm{C}$. F, fetus; $N$, neonate; $A$, adult; $C$, control; $\mathrm{Tx}+\mathrm{T}_{3}, \mathrm{Tx}$ injected with a daily dose of $T_{3}(200 \mu \mathrm{g} / 100$ grams of body weight) and killed at the indicated times after the first injection. 



brains of control and hypothyroid 15-d-old neonates was probed with a rat mitochondrial probe. As shown in Fig. 5, the abundance of mitochondrial DNA was unaltered despite the observed changes in mitochondrial transcripts, indicating that mitochondrial gene expression is regulated by $\mathrm{T}_{3}$ at the level of transcription and/or RNA stabilization and not by gene dosage.
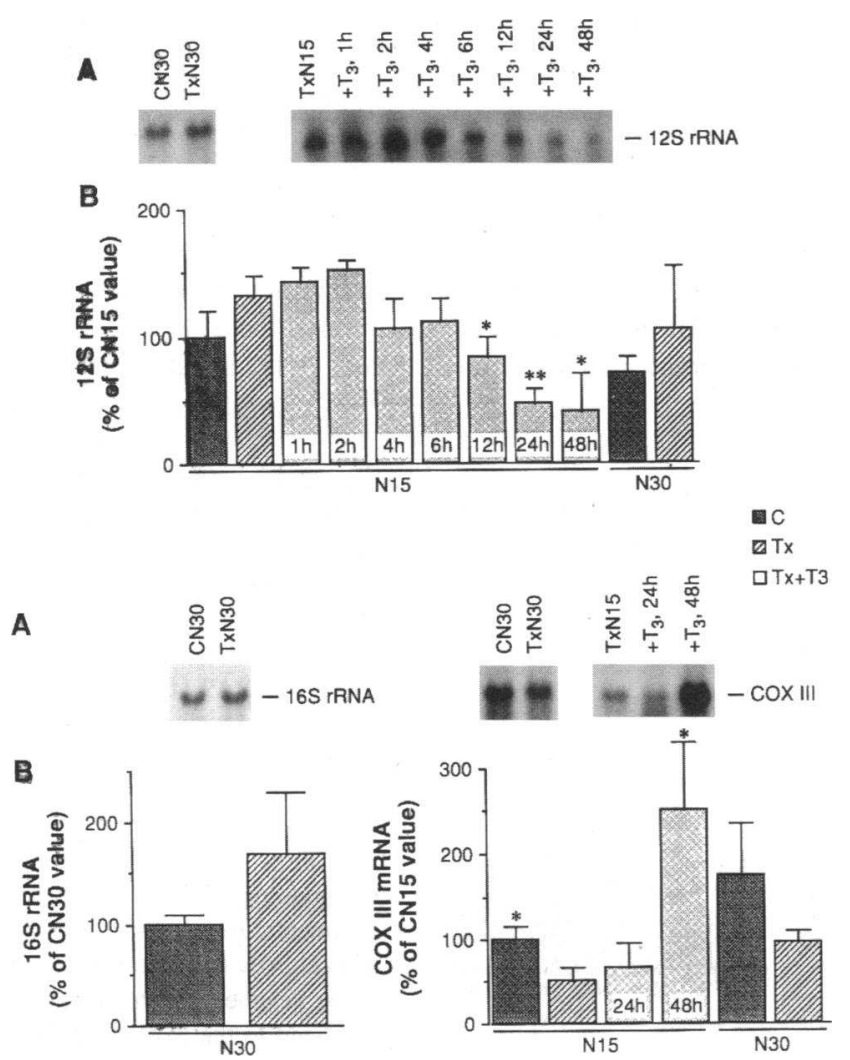

Figure 4. Thyroid hormone regulation of liver mitochondrial genes (12S rRNA, 16S rRNA, and COX III) during development. N, neonate; $\mathrm{C}$, control; $T x+T_{3}, T x$ injected with a daily dose of $T_{3}(200 \mu \mathrm{g} / 100$ grams of body weight) and killed at the indicated times after the first injection. $(A)$ Northern analysis were performed as described in Methods. The autoradiograms shown are representative of three different experiments. $(B)$ Densitometric analysis of three different Northern's corresponding to three different experimental groups. Results are expressed as mean $\pm \mathrm{SD}$. ${ }^{*} P \leq 0.05$ vs $\mathrm{Tx},{ }^{* * P} \leq 0.01$ vs $\mathrm{Tx}$.

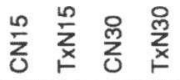

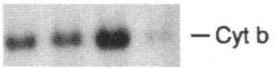

Figure 3. Thyroid hormone regulation of brain mitochondrial genes (COX I, apocytochrome $b$, and ND4) during development. F fetuses; $\mathrm{N}$, neonate; $\mathrm{C}$, control;

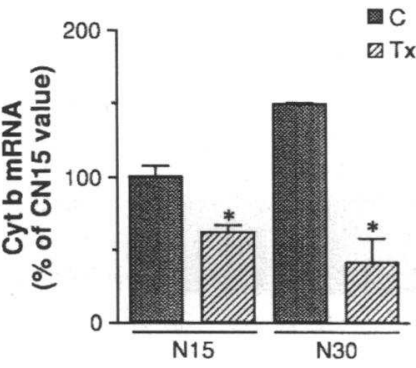

$(A)$ Northern analysis were performed as described in Methods. The autoradiograms shown are representative of three different experiments. $(B)$ Densitometric analysis of three different Northern's corresponding to three different experimental groups. Results are expressed as mean $\pm \mathrm{SD}$. $* P \leq 0.05$ vs $\mathrm{C}$.

Effect of hypothyroidism on the expression of nuclear-encoded mitochondrial sequences. To determine whether there is a coordinated decrease in the expression of both nuclear and mitochondrially encoded transcripts, we measured the levels of mRNAs for two nuclear-encoded subunits of cytochrome $c$ oxidase: subunits IV and VIc, in the brains of control and hypothyroid animals. As shown in Fig. 6, congenital hypothyroidism caused a significant twofold reduction on the mRNA levels for the two tested subunits of cytochrome $c$ oxidase in 6- and 15-d-old neonates. By contrast, no differences were detected in 30-d-old animals. This phenomenon of late normalization of mRNA levels in the brain of hypothyroid animals, has been already observed with other $\mathrm{T}_{3}$-regulated sequences, and is different depending on the brain region studied $(14,28)$. As happened with mitochondrially encoded mitochondrial RNAs, $T_{3}$ treatment to 6-d-old hypothyroid neonates increased the levels of the two nuclear-encoded mitochondrial transcripts studied (data not shown).

Effect of hypothyroidism on brain cytochrome $c$ oxidase activity. Finally, to ensure that the changes in mtRNAs had a functional significance in the mitochondria we also studied the cytochrome $c$ oxidase activity in brains isolated from control and hypothyroid 15-d-old neonates. In keeping with the mtRNA data, we found a significant (40\%) decrease in COX activity in the hypothyroid brain (Table I).

\section{$0.5 \mu \mathrm{g}$}

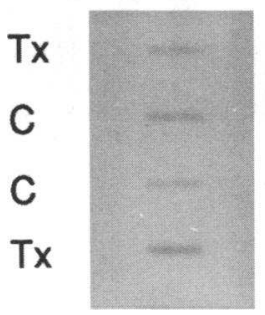

$1 \mu \mathrm{g}$

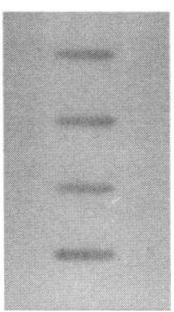

$2 \mu \mathrm{g}$

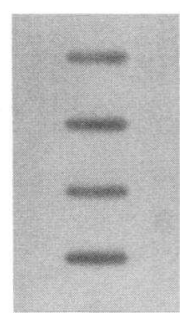

Figure 5. Effect of hypothyroidism on brain mtDNA content. Different amounts of DNA isolated from the brains of 15-d-old control $(C)$ or hypothyroid $(T x)$ animals were loaded onto nylon membranes and hybridized with the cDNA to the 16S rRNA gene. Blots were scanned with a Silver Scanner densitometer and used to measure mitochondrial DNA content. 

mitochondrial genes (COX IV and COX VIc) during develop-
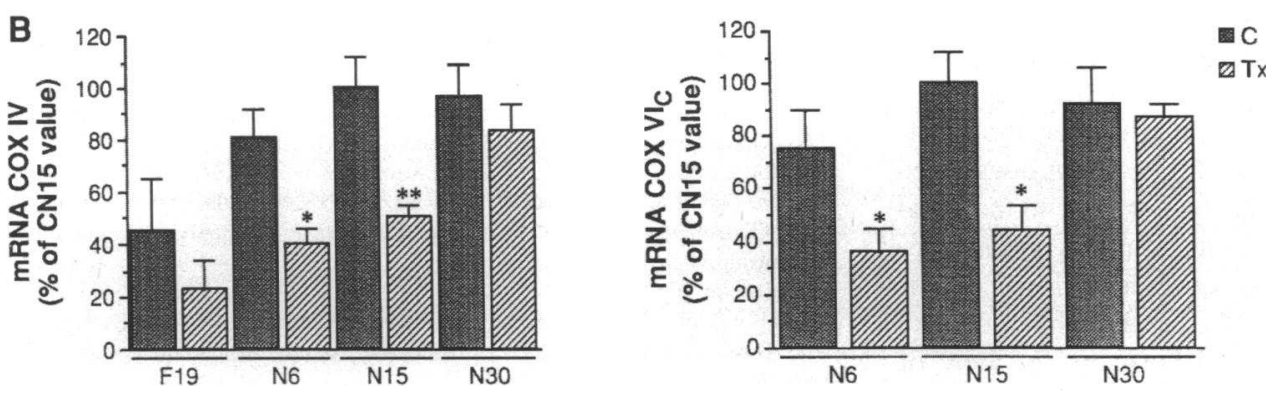
ment. $\mathrm{N}$, neonate; $\mathrm{A}$, adult; $\mathrm{C}$, control. (A) Northern analysis were performed as described in Methods. The autoradiograns shown are representative of three different experiments. ( $B$ ) Densitometric analysis of three different Northern's corresponding to three different experimental groups. Results are expressed as mean $\pm \mathrm{SD}$. $* P \leq 0.05$ vs $\mathrm{C} ; \quad * * P \leq 0.01$ vs C.

\section{Discussion}

The present study clearly demonstrates thyroid hormone regulation of mitochondrial genes during brain development. Thyroid hormone is one of the main regulators of basal metabolic rate in mammals. Due to its stimulatory action on basal metabolism, thyroid hormone increases respiration. However, each tissue responds in a different fashion and whereas a wealth of evidence has been presented that thyroid hormone increases the respiratory rate in liver, heart, and kidney $(16,29)$, there is a great controversy concerning thyroid hormone action on the brain mitochondria. Although there are several reports showing changes in some mitochondrial functions in the brain of hypothyroid animals, studies measuring oxygen consumption have failed to observe any differences between control and hypothyroid brain $(15,16,30)$. Our results clearly show that the lack of $T_{3}$ results in significant changes in the levels of both, nuclearencoded and mitochondrially encoded, mitochondrial RNAs. The level of all the subunits of the cytochrome $c$ oxidase measured are significantly lowered in the brains of 15-d-old hypothyroid animals and, as a consequence, there is a significant reduction in COX activity. These results are in agreement with previous data from Battie et al. (31) showing a diminished activity of the COX enzyme in synaptosomal mitochondria from 14-d-old hypothyroid rats. The above observations also support earlier studies showing an effect of $\mathrm{T}_{3}$ on oxidative phosphorylation (32) as well as morphological alterations of brain mitochondria during neonatal hypothyroidism (33).

Although the targets of $T_{3}$ action on the developing brain

Table I. Effect of Neonatal Hypothyroidism on the Specific Activity of COX Enzyme in the Brains of 15-d-old Neonates

\begin{tabular}{lcc}
\hline & Specific activity & $\begin{array}{c}\text { Percentage of } \\
\text { control value }\end{array}$ \\
\hline & $m U / m g$ protein & \\
$\mathrm{C}$ & $161 \pm 20$ & 100 \\
$\mathrm{Tx}$ & $97 \pm 9$ & 60
\end{tabular}

C, control. The data correspond to the average of at least three determinations in each of two independent experiments. $P<0.002$. are not well known, the importance of adequate levels of thyroid hormone during the perinatal period, has been known for years. However, it was thought that the presence of $T_{3}$ during fetal life was not necessary since, to our knowledge, conclusive evidence regarding a prenatal role of $T_{3}$ in rat brain development is still lacking. In this report we present direct evidence supporting a role of $\mathrm{T}_{3}$ in the development of the central nervous system late in gestation. Our results clearly show a significant decrease of $16 \mathrm{~S}$ and $12 \mathrm{~S}$ rRNAs in the brains of hypothyroid F19 and ND, respectively. In fact, it now appears that thyroid hormones are present in the fetal brain even before endogenous hormone synthesis, and the presence of this hormone as well as its receptors has been shown in rats $(11,34)$ and in humans $(35)$. Our data support the idea that the "critical period" in the rat in which the brain is particularly sensitive to the action of $T_{3}$, originally proposed as beginning of birth, could be extended to the end of pregnancy. On the other hand, since it has been shown that differentiation of mitochondria occurs very rapidly after birth (36), these results suggest that normal mitochondrial maturation is impaired in the brain of hypothyroid neonates.

An interesting observation is the differential regulation by $\mathrm{T}_{3}$ of the different mitochondrial RNAs in the different tissues. Although, in agreement with previous reports in adult rats (27), the levels of COX III transcripts in the liver mitochondria are slightly reduced in the hypothyroid animals and increase after hormone treatment, the concentration of 12S rRNA is significantly lowered in the livers of 15-d-old hypothyroid montes $12 \mathrm{~h}$ after the injection of $\mathrm{T}_{3}$. Whatever the mechanisms underlying this differential regulation, there might be important differences in the regulatory factors of these genes in liver as opposed to brain. In this regard it should be mentioned that thyroid hormone receptors belong to a large family of ligandactivated transcriptional enhancer proteins (5) that regulate gene transcription through a complex combinatorial pattern of interactions between the different members of the family and with other nuclear proteins $(7,37,38)$. Therefore, regulation of these coregulatory proteins in a tissue- and/or temporally specific manner could account for the observed uncoordinated regulation of mtRNAs in brain and liver.

The mechanisms that control mitochondrial gene expression are at present poorly understood. There are several reports showing control at the level of gene dosage, with the amount of mitochondrial transcripts reflecting the content of mtDNA 
per cell (39). However, in cells undergoing differentiation, regulation at transcriptional and translational levels has been described $(40,41)$. Our data showing regulation of all the mitochondrial transcripts tested, together with the lack of differences in the mtDNA content between control and hypothyroid neonates, strongly suggest an effect of thyroid hormone on mitochondrial transcription and/or RNA stabilization. Mitochondrial binding sites for thyroid hormone have been postulated since $1975(42,43)$, and different proteins have been suggested to be the mitochondrial $T_{3}$ receptor (44). However, the specificity of these mitochondrial binding sites has been questioned since then, and specific binding of thyroid hormone to the mitochondria appears to be very questionable. On the contrary, nuclear $\mathbf{T}_{3}$ receptors are very well characterized and, since their discovery in 1972 by Oppenheimer (45), are thought to mediate most of the cellular actions of thyroid hormone. In view of these evidences, and taking into account the delayed kinetic response to $T_{3}$ of brain mitochondrial transcripts, it is very probable that $T_{3}$ action on mitochondrial transcription is exerted trough its nuclear receptors.

To date, the genetic control of mitochondrial function is largely unknown. Several nuclear genes for proteins involved in mitochondrial transcription have been cloned, including those for a human mitochondrial transcription factor (h-mtTFA) (46, 47 ), the human and mouse genes for the RNA subunit of mitochondrial RNA-processing endonuclease (48), a ribonucleoprotein enzyme that is thought to cleavage light-strand transcripts to form primers for heavy-strand DNA replication, and the yeast mtRNA polymerase (49). It has also been cloned a human gene coding for a protein designated as nuclear respiratory factor-1 (NRF-1), which plays a role in the coordinate expression of nuclear-encoded respiratory chain subunits (50). Little is known however of the transcription factors controlling rat mitochondrial transcription. Therefore, the understanding of the observed $T_{3}$ effects on brain mitochondria will pass for the identification of these proteins as well as a profound study of their regulation by $T_{3}$.

In summary, our results support the concept that $\mathbf{T}_{3}$ participates in a signal transduction pathway that regulates mitochondrial function in the central nervous system. Although a direct effect of $T_{3}$ on mtDNA can not be completely excluded, the lack of conclusive evidence for the presence of $T_{3}$ receptors in the mitochondria suggest that a hormone-induced signal from the nucleus crosses the mitochondrial membrane and is responsible for the regulated expression of the mitochondrial genome. Whether the exact locus of regulation is transcription and/or RNA stabilization, and how the information for this regulation is transmitted from the nucleus to the mitochondria, remains to be determined. In any case, the direct evidence presented in this paper showing the effect of $T_{3}$ on brain mitochondrial gene expression is particularly important on the light of the growing evidence that a broad spectrum of diseases affecting the central nervous system is associated with alterations in mitochondrial function (51). Since the absence of $T_{3}$ in the neonatal period also produces an important impairment of brain functions, our results supply one explanation in molecular terms to understand the phenotypic effect observed in hypothyroid brain.

\section{Acknowledgments}

We wish to thank Dr. P. Cantatore for the generous gift of rat mtDNA clones apocytochrome $b$, ND4 and COX I. The expert technical assistant of $\mathrm{P}$. Ochoa is fully acknowledged.
E. Vega-Núñez is a predoctoral fellow from the Comunidad de Madrid. This research was supported by the Fondo de Investigaciones Sanitarias de la Seguridad Social Grants 92/0280 (A. Perez-Castillo), 95/0896 (A. Perez-Castillo), 94/0284 (A. Santos), and 93/0339 (R. Garesse) and by the Comunidad de Madrid Grant C170/91 (A. PerezCastillo).

\section{References}

1. Oppenheimer, J. H., H. L. Schwartz, C. N. Mariash, W. B. Kinlaw, W. C. W. Wong, and H. C. Freake. 1987. Advances in our understanding of thyroid hormone action at the cellular level. Endocr. Rev. 8:288-308.

2. Oppenheimer, J. H. 1983. The nuclear receptor-triiodothyronine complex: relationship to thyroid hormone distribution, metabolism, and biological action. In Molecular Basis of Thyroid Hormone Action. J. H. Oppenheimer and H. H. Samuels, editors. Academic Press Inc., New York. 1-35.

3. Dussault, J. H., and J. Ruel. 1987. Thyroid hormones and brain development. Annu. Rev. Physiol. 49:321-334.

4. Legrand, J. 1986. Thyroid hormone effects on growth and development. In Thyroid Hormone Metabolism. G. Henneman, editor. Marcel Decker, Inc., New York. 503-534.

5. Evans, R. 1988. The steroid and thyroid hormone receptor superfamily. Science (Wash. DC). 240:889-895.

6. Saatcioglu, F., T. Deng, and M. Karin. 1993. A novel cis element mediating ligand-independent activation by c-ErbA: Implications for hormonal regulation. Cell. 75:1095-1105.

7. Yu, V. C., C. Delsert, B. Andersen, J. M. Holloway, O. V. Devary, A. M. Näär, S. Y. Kim, J. M. Boutin, C. K. Glass, and M. G. Rosenfeld. 1991. RXR $\beta$ : a coregulator that enhances binding of retinoic acid, thyroid hormone and vitamin D receptors to their cognate response elements. Cell. 67:1251-1266.

8. Schwartz, H. L. 1983. Effect of thyroid hormone in growth and development. In Molecular Basis of Thyroid Hormone Action. J. H. Oppenheimer and H. H. Samuels, editors. Academic Press Inc., New York. 413-444.

9. Samuels, H. H. B. M. Forman, Z D. Horowitz, and Z S. Ye 1988. Regulation of gene expression by thyroid hormone. J. Clin. Invest. 81:957-967.

10. Oklund, S., and P. S. Timiras. 1977. Influences of thyroid levels in brain ontogenesis in vivo and in vitro. In Thyroid Hormone and Brain Development. G. D. Grave, editor. Raven Press, Ltd., New York. 33-47.

11. Perez-Castillo, A., J. Bernal, B. Ferreiro, and T. Pans. 1985. The early ontogenesis of thyroid hormone receptor in the rat. Endocrinology. 117:24572461 .

12. Strait, K. A., H. L. Schwartz, A. Perez-Castillo, and J. H. Oppenheimer 1990. Relationship of c-erbA content to tissue triiodothyronine nuclear binding capacity and function in developing and adult rats. J. Biol. Chem. 265:1051410521.

13. Pipaón, C., A. Santos, and A. Perez-Castillo. 1992. Thyroid hormone upregulates NGFI-A gene expression in rat brain during development. J. Biol. Chem. 267:21-23.

14. Mellström, B., C. Pipaón, J. R. Naranjo, A. Perez-Castillo, and A. Santos. 1994. Differential effect of thyroid hormone on NGFI-A gene expression in developing rat brain. Endocrinology 135:583-588.

15. Schwartz, H. L., and J. H. Oppenheimer. 1978. Ontogenesis of 3,5,3'triiodothyronine receptors in neonatal rat brain: dissociation between receptor concentration and stimulation of oxygen consumption by 3,5,3'-triiodothyronine. Endocrinology 103:943-948.

16. Satav, J. G., and S. S. Katyare. 1982. Effect of experimental thyrotoxicosis on oxidative phosphorylation in rat liver, kidney and brain mitochondria. $\mathrm{Mol}$. Cell. Endocrinol. 28:173-189.

17. Pruitt, S. C. 1988. Expression vectors permitting cDNA cloning and enrichment for specific sequences by hybridization/selection. Gene 66:121-134.

18. Chomcynsky, P., and N. Sacchi. 1986. Single-step method of RNA isolation by acid guanidinium thiocyanate-phenol-chlorophorm extraction. Anal. Biochem. 162:156-159.

19. Maniatis, T., E. F. Fritsh, and K. J. Sambrook. 1982. Molecular cloning. A laboratory manual. Cold Spring Harbor Laboratory, Cold Spring Harbor, NY. 280-281.

20. Vallejo, C. G., M. A. G. Sillero, and R. Marco. 1979. Mitochondrial maturation during Artemia Salina embryogenesis. General description of the process. Cell Mol. Biol. 25:113-124.

21. Bradford, M. M. 1976. A rapid and sensitive method for the quantitation of microgram quantities of protein utilizing the principle of protein-dye binding. Anal. Biochem. 72:248-254.

22. Legrand, J. 1979. Morphogenetic actions of thyroid hormones. Trends Neurosci. 2:234-236.

23. Porterfield, S. P., and C. E. Hendrich. 1993. The role of thyroid hormones in prenatal and neonatal neurological development. Current perspectives. Endocr. Rev. 14:94-106. 
24. Tzagoloff, A., and A. M. Myers. 1986. Genetics of mitochondrial biogenesis. Annu. Rev. Biochem. 55:249-285.

25. Mutvei, A., S. Kuzela, and B. D. Nelson. 1989. Control of mitochondrial transcription by thyroid hormone. Eur. J. Biochem. 180:235-240.

26. Garstka, H. L., M. Fäcke, J. R. Escribano, and R. J. Wiesner. 1994. Stoichiometry of mitochondrial transcripts and regulation of gene expression by mitochondrial transcription factor A. Biochem. Biophys. Res. Commun. 200:619626.

27. Wiesner, R. J., T. T. Kurowski, and R. Zak. 1992. Regulation by thyroid hormone of nuclear and mitochondrial genes encoding subunits of cytochrome$c$-oxidase in rat liver and skeletal muscle. Mol. Endocrinol. 6:1458-1467.

28. Strait, K. A., L. Zou, and J. H. Oppenheimer. 1992. $\beta 1$-Isoform-specific regulation of a triiodothyronine-induced gene during cerebellar development. Mol. Endocrinol. 6:1874-1880.

29. Larsen, P. R., and S. H. Ingbar. 1992. The thyroid gland. In Textbook of Endocrinology. M. O. Thorner, M. L. Vance, and K. Kovacks, editors. Harcourt Brace Jovanovich Inc., Philadelphia. 357-489.

30. Gomez, C. J., A. J. De Guglielmone, and B. Duvilanski. 1971. Effect of neonatal thyroidectomy on ribonucleic acid synthesis in developing rat brain. Acta Physiol. Lat. Am. 21:152-155.

31. Battie, C. A., and M. A. Verity. 1979. Membrane enzyme development in nerve ending mitochondria during neonatal hypothyroidism. Dev. Neurosci. 2:139-148.

32. Katyare, S. S., M. V. Joshi, P. Fatterpaker, and A. Screenivasan. 1977. Effect of thyroid deficiency on oxidative phosphorylation in rat liver, kidney and brain mitochondria. Arch. Biochem. Biophys. 182:155-163.

33. Gadaleta, M. N., M. Renis, G. R. Minervini, I. Serra, T. Bleve, A. Giovine, G. Zacheo, and Giuffrida. 1985. Effect of hypothyroidism on the biogenesis of free mitochondria in the cerebral hemispheres and in the cerebellum of rat during postnatal development. Neurochem. Res. 10:163-177.

34. Obregon, M. J., J. Mallol, R. Pastor, G. Morreale de Escobar, and F. Escobar del Rey. 1984. L-thyroxine and 3,5,3'-triiodo-L-thyronine in rat embryos before onset of fetal thyroid function. Endocrinology. 114:305-307.

35. Bernal, J., A. Perez-Castillo, A. Pans, and F. Pekonen. 1984. Ontogenesis of thyroid hormone receptor. In Endocrinology: International Congress Series 655. L. Labrie and L. Prouxl, editors. Excerpta Medica, Amsterdam. 977-981.

36. Izquierdo, J. M., A. M. Luis, and J. M. Cuezva. 1990. Postnatal mitochondrial differentiation in rat liver. Regulation by thyroid hormones of the $\beta$-subunit of the mitochondrial F1-ATPase complex. J. Biol. Chem. 265:9090-9097.

37. Zhang, X.-K., B. Hoffman, P. B.-V. Tran, G. Graupner, and M. Pfahl.
1992. Retinoid $X$ receptor is an auxiliary proten for thyroid hormone and retinoic acid receptors. Nature (Lond.). 355:441-446.

38. Desbois, C., D. Aubert, C. Legrand, B. Pain, and J. Samarut. 1991. A novel mechanism of action for v-erbA: abrogation of the inactivation of transcription factor AP-1 by retinoic acid and thyroid hormone receptors. Cell. 67:731740.

39. William, R. S. 1986. Mitochondrial gene expression in mammalian striated muscle. J. Biol. Chem. 261:12390-12394.

40. Pikó, L., and K. D. Taylor. 1987. Amounts of mitochondrial DNA and abundance of some mitochondrial gene transcripts in early mouse embryos. Dev. Biol. 123:364-374.

41. Luis, A. M., J. M. Izquierdo, L. K. Ostronoff, M. Salinas, J. F. Santaren, and J. M. Cuezva. 1993. Translational regulation of mitochondrial differentiation in neonatal rat liver. Specific increase in the translational efficiency of the nuclearencoded mitochondrial beta-F1-ATPase mRNA. J. Biol. Chem. 268:1868-1875.

42. Sterling, K., and P. O. Milch. 1975. Thyroid hormone binding by a component of mitochondrial membrane. Proc. Natl. Acad. Sci. USA. 72:3225-3229.

43. Sterling, K., G. A. Campbell, and M. A. Brenner. 1984. Purification of the mitochondrial triiodothyronine (T3) receptor from rat liver. Acta Endocrinol. 105:391-397.

44. Sterling, K. 1986. Direct thyroid hormone activation of mitochondria: the role of adenine nucleotide translocase. Endocrinology. 119:292-295.

45. Oppenheimer, J. H., D. Koerner, H. L. Schwartz, and M. I. Surks. 1972. Specific nuclear triiodothyronine binding sites in rat liver and kidney. J. Clin. Endocrinol. Metab. 35:330-333.

46. Xu, B., and D. A. Clayton. 1992. Assignment of a yeast protein nocessary for mitochondrial transcription initiation. Nucleic Acids Res. 20:1053-1059.

47. Fiher, R. P., J. N. Topper, and D. A. Clayton. 1987. Promoter selection in human mitochondria involves binding of a transcription factor to orientationindependent upstream regulatory elements. Cell. 50:247-258.

48. Topper, J. N., and D. A. Clayton. 1990. Characterization of human MRP/ Th RNA and its nuclear gene: Full length MRP/Th RNA is an active endoribonuclease when assembled as an RNP. Nucleic Acids Res. 18:793-799.

49. Masters, B. S., L. L. Stohl, and D. A. Clayton. 1987. Yeast mitochondrial RNA polymerase is homologous to those encoded by bacteriophages T3 and $\mathrm{T} 7$. Cell. 51:89-99.

50. Virbasius, C. A., J. V. Virbasius, and R. C. Scarpulla. 1993. NRF-1, an activator involved in nuclear-mitochondrial interactions, utilizes a new DNAbinding domain conserved in a family of developmental regulators. Genes \& Dev. 7:2431-2445.

51. Schapira, A. H. V. 1993. Mitochondrial cytopathies. Curr. Opin. Newrobiol. 3:760-767. 